\title{
On I-Limit Superior and I-Limit Inferior of Sequences in Intuitionistic Fuzzy Normed Spaces
}

\author{
Mausumi Sen \\ NIT Silchar \\ Silchar 788010, India
}

\begin{abstract}
In this article we introduce the notions of $I$-limit superior and $I$-limit inferior for sequences in intuitionistic fuzzy normed linear spaces and prove intuitionistic fuzzy analogue of some results of $I$-limit superior and $I$-limit inferior for real sequences. The concept of $I$-limit points and $I$-cluster points in intuitionistic fuzzy normed linear spaces are introduced and some of their properties have been established.
\end{abstract}

\section{Keywords}

Intuitionistic fuzzy normed linear space, I-convergence, Ilimit superior, $I$-limit inferior

\section{INTRODUCTION}

Since the introduction of fuzzy set theory by Zadeh [9] in 1965, fuzzy logic became an important area of research in various branches of mathematics such as metric and topological spaces [4], theory of functions [5], approximation theory [1], etc. Fuzzy set theory also finds its applications for modeling uncertainty and vagueness in various fields of science and engineering, e.g. computer programming, nonlinear dynamical systems, population dynamics, control of chaos, etc. The theory of intuitionistic fuzzy set, introduced by Atanassov [2], is a very natural tool for modeling imprecision in real life situations and found its application in various area of science and engineering. Saadati and Park [7] introduced the notion of intuitionistic fuzzy normed linear space.

The notion of $I$-convergence of real valued sequence was studied at the initial stage by Kostyrko, Šalát and Wilczyński [6]. It generalizes and unifies different notions of convergence of sequences. Generalizing the concepts of limit superior and limit inferior for real sequences, Demirci [3] introduced the concepts of $I$-limit superior and $I$-limit inferior for sequences of real numbers.

The aim of the present paper is to introduce and investigate Ilimit superior and $I$-limit inferior for sequences in intuitionistic fuzzy normed linear space and obtain some important results on them. We also introduce the idea of $I$ limit points and $I$-cluster points in intuitionistic fuzzy normed linear space and study their properties.

\section{PRELIMINARIES}

Throughout this paper $\mathrm{N}$ and $\mathrm{R}$ will denote the sets of natural numbers and real numbers respectively. The definitions of continuous t-norm and continuous t-conorm can be found in [8].

Definition 2.1. An intuitionistic fuzzy normed linear space (in short, IFNLS) is a five-tuple $(\mathrm{X}, \mu, v, *, o)$, where $\mathrm{X}$ is a linear space, $*$ is a continuous $\mathrm{t}$-norm, $\mathrm{o}$ is a continuous $\mathrm{t}$ conorm and $\mu, v$ are fuzzy sets on $X \times(0, \infty)$ satisfying the following conditions for every $\mathrm{x}, \mathrm{y} \in \mathrm{X}$ and $\mathrm{s}, \mathrm{t}>0$ :

(i) $\mu(x, t)+v(x, t) \leq 1$,

(ii) $\mu(x, t)>0$, (iii) $\mu(x, t)=1$ if and only if $x=0$,

(iv) $\mu(\alpha \mathrm{x}, \mathrm{t})=\mu\left(\mathrm{x}, \frac{t}{|\alpha|}\right)$ for each $\alpha \neq 0$,

(v) $\mu(\mathrm{x}, \mathrm{t}) * \mu(\mathrm{y}, \mathrm{s}) \leq \mu(\mathrm{x}+\mathrm{y}, \mathrm{t}+\mathrm{s})$,

(vi) $\mu(\mathrm{x}, \mathrm{t}):(0, \infty) \rightarrow[0,1]$ is continuous in $\mathrm{t}$,

(vii) $\lim _{t \rightarrow \infty} \mu(\mathrm{x}, \mathrm{t})=1$ and $\lim _{t \rightarrow 0} \mu(\mathrm{x}, \mathrm{t})=0$,

(viii) $v(x, t)<1$,

(ix) $v(x, t)=0$ if and only if $x=0$,

(x) $v(\alpha x, t)=v\left(x, \frac{t}{|\alpha|}\right)$ for each $\alpha \neq 0$,

(xi) $v(\mathrm{x}, \mathrm{t})$ o $v(\mathrm{y}, \mathrm{s}) \geq \mathrm{v}(\mathrm{x}+\mathrm{y}, \mathrm{t}+\mathrm{s})$,

(xii) $v(x, t):(0, \infty) \rightarrow[0,1]$ is continuous in $t$,

(xiii) $\lim _{t \rightarrow \infty} \mathrm{v}(\mathrm{x}, \mathrm{t})=0$ and $\lim _{t \rightarrow 0} \mathrm{v}(\mathrm{x}, \mathrm{t})=1$.

Definition 2.2. Let $(X, \mu, v, *, o)$ be an IFNLS. A sequence $\mathrm{x}$ $=\left({ }^{x_{k}}\right)$ in $\mathrm{X}$ is said to be convergent to $\mathrm{L} \in \mathrm{X}$ with respect to the intuitionistic fuzzy norm $(\mu, v)$ if, for every $\varepsilon>0$ and $t>0$, there exists $k_{0} \in \mathrm{N}$ such that $\mu\left(x_{k}-\mathrm{L}, \mathrm{t}\right)>1-\varepsilon$ and $v\left(x_{k}-\mathrm{L}\right.$, t) $<\varepsilon$ for all $\mathrm{k} \geq k_{0}$. It is denoted by $(\mu, v)-\lim \mathrm{x}=\mathrm{L}$.

Definition 2.3. Let $X$ be a non empty set. A non-void class I $\subseteq \mathrm{P}(\mathrm{X})$ (power set of $\mathrm{X}$ ) is called an ideal if $\mathrm{I}$ is additive (i.e. $\mathrm{A}, \mathrm{B} \in \mathrm{I} \Rightarrow \mathrm{A} \cup \mathrm{B} \in \mathrm{I}$ ) and hereditary (i.e. $\mathrm{A} \in \mathrm{I}$ and $\mathrm{B} \subseteq \mathrm{A} \Rightarrow$ $\mathrm{B} \in \mathrm{I})$.

For each ideal $\mathrm{I}$ there is a filter $=\mathrm{F}(\mathrm{I})$ corresponding to $\mathrm{I}$, given by

$$
F(I)=\{K \subseteq N: M K \in I\} .
$$

Definition 2.4. A sequence $\mathrm{x}=\left({ }^{x_{k}}\right)$ of numbers is $\mathrm{I}$ convergent to ${ }^{x_{0}}$ if for every $\varepsilon>0$, the set $\left\{\mathrm{k} \in \mathrm{N}:\left.\right|^{x_{k}}{ }^{x_{0}} \mid \geq\right.$ $\varepsilon\} \in \mathrm{I}$ and we write $\mathrm{I}-\operatorname{limx}=x_{0}$.

Definition 2.5. Let $I \subseteq P(N)$ and $(X, \mu, v, *, o)$ be an IFNLS. A sequence $\mathrm{x}=\left(x_{k}\right)$ in $X$ is said to be $I$-convergent to $L \in X$ with respect to the intuitionistic fuzzy norm $(\mu, v)$ if, for every $\varepsilon>0$ and $t>0$ the set $\left\{k \in N: \mu\left(x_{k}-L, t\right) \leq 1-\varepsilon\right.$ or $v\left(x_{k}-L, t\right)$ $\geq \varepsilon\} \in I$ and we write $I_{(\mu, v)}-\lim x=L$. 
Definition 2.6. A sequence $x=\left(x_{k}\right)$ of numbers is said to be $I$ bounded with respect to the intuitionistic fuzzy norm $(\mu, v)$ if there exists some $t_{0} \in R$ and $\varepsilon>0$ such that $\left\{k \in N: \mu\left(x_{k}\right.\right.$, $\left.t_{0}\right) \leq 1-\varepsilon$ or $\left.v\left(x_{k}, t_{0}\right) \geq \varepsilon\right\} \in I$.

\section{I-LIMIT SUPERIOR AND I-LIMIT INFERIOR IN IFNLS}

In this section we introduce the notions of $I$-limit superior and $I$-limit inferior for sequences in intuitionistic fuzzy normed linear spaces.

Definition 3.1. For a sequence $x$ in IFNLS, let us define the sets

$B_{x}^{(\mu, v)}=\left\{b \in(0,1):\left\{k \in N: \mu\left(x_{k}, t\right)<1-b\right.\right.$ or $v\left(x_{k}, t\right)>$ $b\} \notin I\}$

$A_{x}^{(\mu, v)}=\left\{a \in(0,1): \quad\left\{k \in N: \mu\left(x_{k}, t\right)>1-a\right.\right.$ or $v\left(x_{k}, t\right)<$ $a\} \notin I\}$

Then the $I$-limit superior of $x$ with respect to the intuitionistic fuzzy norm $(\mu, v)$ is defined by

$I_{(\mu, v)}-\lim \sup x=\left\{\begin{array}{cccc}\sup B_{x}^{(\mu, v)}, & \text { if } & B_{x}^{(\mu, v)} \neq \phi \\ 0, & \text { if } & B_{x}^{(\mu, v)}=\phi .\end{array}\right.$

Also the I-limit inferior of $x$ with respect to the intuitionistic fuzzy norm $(\mu, v)$ is defined by

$I_{(\mu, v)}-\liminf x=\left\{\begin{array}{cccc}\inf A_{x}^{(\mu, v)}, & \text { if } & A_{x}^{(\mu, v)} \neq \phi \\ 1, & \text { if } & A_{x}^{(\mu, v)}=\phi .\end{array}\right.$

Theorem 3.2. If $b=I_{(\mu, v)}-\lim \sup x$, then for every positive numbers $t$ and $\varepsilon$

$\left\{k \in N: \mu\left(x_{k}, t\right)<1-b+\varepsilon\right.$ or $\left.v\left(x_{k}, t\right)>b-\varepsilon\right\} \notin I$ and

$\left\{k \in N: \mu\left(x_{k}, t\right)<1-b-\varepsilon\right.$ or $\left.v\left(x_{k}, t\right)>b+\varepsilon\right\} \in I$.

Conversely if (1) holds for every positive $\varepsilon$ and $t$, then $b=$ $I_{(\mu, v)}-\lim \sup x$.

Proof. Let $b=I_{(\mu, v)}-\lim \sup x$, where $b$ is finite. Then the set

$$
K=\left\{k \in N: \mu\left(x_{k}, t\right)<1-b \text { or } v\left(x_{k}, t\right)>b\right\} \notin I .
$$

Since $\mu\left(x_{k}, t\right)<1-b+\varepsilon$ or $v\left(x_{k}, t\right)>b-\varepsilon$, for every $k \in K$ and for any $t, \varepsilon>0$, the set

$$
\left\{k: \mu\left(x_{k}, t\right)<1-b+\varepsilon \text { or } v\left(x_{k}, t\right)>b-\varepsilon\right\} \notin I .
$$

Now from the definition of $I_{(\mu, v)}-\lim \sup x$, we have $1-b$ is the least value and $b$ is the greatest value satisfying (2). Now, if possible, let

$$
\left\{k: \mu\left(x_{k}, t\right)<1-b-\varepsilon \text { or } v\left(x_{k}, t\right)>b+\varepsilon\right\} \notin I .
$$

Then $1-b-\varepsilon$ and $b+\varepsilon$ are another values with $1-b-\varepsilon<1-b$ and $b+\varepsilon>b$ which satisfy (2). This contradicts the fact that
1- $b$ and $b$ are the least and greatest values respectively satisfying the above condition.

Hence, $\left\{k \in N: \mu\left(x_{k}, t\right)<1-b-\varepsilon\right.$ or $\left.v\left(x_{k}, t\right)>b+\varepsilon\right\} \in I$, for every $\varepsilon>0$.

Conversely, if (1) holds for every $t, \varepsilon>0$, then

$$
\left\{k \in N: \mu\left(x_{k}, t\right) \leq 1-b \text { or } v\left(x_{k}, t\right) \geq b\right\} \notin I
$$

and $\left\{k \in N: \mu\left(x_{k}, t\right)=1-b\right.$ or $\left.v\left(x_{k}, t\right)=b\right\} \in I$.

Thus $\left\{k \in N: \mu\left(x_{k}, t\right)<1-b\right.$ or $\left.v\left(x_{k}, t\right)>b\right\} \notin I$, for every $t>0$.

Hence $b=I_{(\mu, v)}-\lim \sup x$.

The similar result for $I_{(\mu, v)}$ - lim inf $x$ can also be proved.

Theorem 3.3. If $a=I_{(\mu, v)}-\lim \inf x$ is finite, then for every positive number $t$ and $\varepsilon$,

$$
\left\{k \in N: \mu\left(x_{k}, t\right)>1-a-\varepsilon \text { or } v\left(x_{k}, t\right)<a+\varepsilon\right\} \notin I
$$

and $\left\{k \in N: \mu\left(x_{k}, t\right)>1-a+\varepsilon\right.$ or $\left.v\left(x_{k}, t\right)<a-\varepsilon\right\} \in I$.

Conversely if (3) holds for every positive $t$ and $\varepsilon$, then $a=$ $I_{(\mu, v)}-\lim \inf x$.

Theorem 3.4. For any sequence $x$,

$$
I_{(\mu, v)}-\lim \inf x \leq I_{(\mu, v)}-\lim \sup x .
$$

Proof. First we consider the case in which $I_{(\mu, v)}-\lim \sup x=$ 0 , which implies that $B_{x}^{(\mu, v)}=\phi$.

Then for $b \in(0,1)$,

$$
\left\{k: \mu\left(x_{k}, t\right)<1-b \text { or } v\left(x_{k}, t\right)>b\right\} \in I .
$$

This implies that $\left\{k: \mu\left(x_{k}, t\right) \geq 1-b\right.$ or $\left.v\left(x_{k}, t\right) \leq b\right\} \in F(I)$. Hence for every $a \in(0,1)$, we have $\left\{k: \mu\left(x_{k}, t\right)>1-a\right.$ or $\left.v\left(x_{k}, t\right)<a\right\} \notin I$ and so $I_{(\mu, v)}-\lim \inf x=0$.

The case in which $I_{(\mu, v)}-\lim \sup x=1$ is trivial.

Now we assume that $b=I_{(\mu, v)}-\lim \sup x$ and $a=I_{(\mu, v)}-\lim$ $\inf x$, where $a$ and $b$ are finite.

For given any $\varepsilon$, we now show that $1-b-\varepsilon \in A_{x}^{(\mu, v)}$.

By theorem 1, we have

$\left\{k \in N: \mu\left(x_{k}, t\right)<1-b-\varepsilon / 2\right.$ or $\left.v\left(x_{k}, t\right)>b+\varepsilon / 2\right\} \in I$.

Therefore, $\left\{k \in N: \mu\left(x_{k}, t\right) \geq 1-b-\varepsilon / 2\right.$ or $v\left(x_{k}, t\right) \leq b+$ $\varepsilon / 2\} \in F(I)$ which implies that

$\left\{k: \mu\left(x_{k}, t\right)>1-b-\varepsilon\right.$ or $\left.v\left(x_{k}, t\right)<b+\varepsilon\right\} \notin I$.

Hence, $1-b-\varepsilon \in A_{x}^{(\mu, \nu)}$.

Since $a=\inf A_{x}^{(\mu, \nu)}$ and so we conclude that $1-b-\varepsilon \leq 1-a$.

Since $\varepsilon$ is arbitrary, we have, $1-b \leq 1-a$ i.e. $\mathrm{a} \leq \mathrm{b}$.

Theorem 3.5. In a IFNLS $(X, \mu, v, *, o)$, if the $I$-bounded sequence $x$ is $I$-convergent then $I_{(\mu, v)}-\lim \inf x=I_{(\mu, v)}$ $\lim \sup x$. 
Proof. Let $I_{(\mu, v)}-\lim \inf x=\alpha$ and $I_{(\mu, v)}-\lim \sup x=\beta$.

Now we assume that $I_{(\mu, v)}-\lim x=L$. Then for every $t>0$ and $b \in(0,1)$, the set

$\left\{k: \mu\left(x_{k}-L, t\right) \leq 1-b\right.$ or $\left.v\left(x_{k}-L, t\right) \geq b\right\} \in I$ so that

$\left\{k: \mu\left(x_{k}, t / 2\right) * \mu(L, t / 2) \leq 1-b\right.$ or $\left.v\left(x_{k}, t / 2\right) \circ v(L, t / 2) \geq b\right\}$

$\in I$.

Let $\sup \mu\left(x_{k}, t / 2\right)=1-b_{1}$ and $\sup \mu(L, t / 2)=1-b_{2}$

or inf $v\left(x_{k}, t / 2\right)=b_{1}$ and $\inf _{t} v(L, t / 2)=b_{2}$

such that $\left(1-b_{1}\right) *\left(1-b_{2}\right) \leq 1-b$ or $b_{1}$ o $b_{2} \geq b$.

Then the set

$\left\{k: \mu\left(x_{k}, t / 2\right) \leq 1-b_{1}\right.$ or $\left.v\left(x_{k}, t / 2\right) \geq b_{1}\right\} \in I$

and so

$\left\{k: \mu\left(x_{k}, t / 2\right) \leq 1-b_{1}-\varepsilon\right.$ or $\left.v\left(x_{k}, t / 2\right) \geq b_{1}+\varepsilon\right\} \in I$, for all $\varepsilon>0$.

Now applying the theorem 1 and the definition of $I_{(\mu, v)}$ - lim $\sup x$, we get

$\left\{k: \mu\left(x_{k}, t / 2\right) \leq 1-\beta-\varepsilon\right.$ or $\left.v\left(x_{k}, t / 2\right) \geq \beta+\varepsilon\right\} \in I, \quad$ for all $\varepsilon>0$.

From (6) and (7) and by the definition of $I_{(\mu, v)}$ - lim supx, we get

$$
1-b_{1}-\varepsilon \leq 1-\beta-\varepsilon \text {. }
$$

Therefore, $\quad \beta \leq b_{1}$.

Now we find those $k$ such that

$$
\mu\left(x_{k}, t / 2\right)>1-b_{1}+\varepsilon \text { or } v\left(x_{k}, t / 2\right)<b_{1}-\varepsilon .
$$

We can easily observe that no such $k$ exists which satisfy (4) together with the above condition. So the set

$$
\left\{k: \mu\left(x_{k}, t / 2\right)>1-b_{1}+\varepsilon \text { or } v\left(x_{k}, t / 2\right)<b_{1}-\varepsilon\right\} \in I .
$$

Since $\alpha=I_{(\mu, v)}-\lim \inf x$, we get

$$
\left\{k: \mu\left(x_{k}, t / 2\right)>1-\alpha+\varepsilon \text { or } v\left(x_{k}, t / 2\right)<\alpha-\varepsilon\right\} \in I .
$$

By the definition of $I_{(\mu, v)}-\lim \inf x$, we have

$$
1-\alpha+\varepsilon \leq 1-b_{1}+\varepsilon \text {. }
$$

Therefore, $b_{1} \leq \alpha$.

Hence from (8) and (9), we get $\beta \leq \alpha$.

Now combining the theorem 3.4 and the above inequality, we conclude that $\alpha=\beta$.

This completes the proof

\section{I-LIMIT POINTS AND I-CLUSTER} POINTS IN IFNLS

Definition 4.1. Let $(X, \mu, v, *, o)$ be a IFNLS. Then $l \in \mathrm{X}$ is said to be a limit point of the sequence $x=\left(x_{k}\right)$ with respect to the intuitionistic fuzzy norm $(\mu, v)$ provided there is a subsequence of $x$ that converges to $l$ with respect to the intuitionistic fuzzy norm $(\mu, v)$. We denote the set of all limit points of the sequence $x$ by $L_{(\mu, v)}(x)$.

Definition 4.2. Let $(X, \mu, v, *, o)$ be a IFNLS. Then $\xi \in X$ is called a $I$ - limit point of the sequence $x=\left(x_{k}\right)$ with respect to the intuitionistic fuzzy norm $(\mu, v)$, provided there exists a set $M=\left\{m_{1}<m_{2}<\ldots\right\} \subset N$ such that $M \notin I$ and $(\mu, v)-\lim$ $x_{m_{k}}=\xi$. Let $\Lambda_{(\mu, v)}^{I}(x)$ denotes the set of all $I$-limit points of the sequence with respect to the intuitionistic fuzzy norm ( $\mu$, $v$ ).

Definition 4.3. Let $(X, \mu, v, *, o)$ be a IFNLS. Then $\gamma \in X$ is called a $I$ - cluster point of the sequence $x=\left(x_{k}\right)$ with respect to the intuitionistic fuzzy norm $(\mu, v)$ provided that for each $\varepsilon>0$ and $\lambda \in(0,1)$, the set $\left\{k \in N: \mu\left(x_{k}-\gamma, \varepsilon\right)>1-\lambda\right.$ and $\left.v\left(x_{k}-\gamma, \varepsilon\right)<\lambda\right\} \notin I$.

Let $\Gamma_{(\mu, v)}^{I}(x)$ denotes the set of all such $I$-cluster points of $x$. Theorem 4.4. Let $(X, \mu, v, *, o)$ be a IFNLS. Then for any sequence $x \in X, \Lambda_{(\mu, v)}^{I}(x) \subseteq \Gamma_{(\mu, v)}^{I}(x)$.

Proof. Let $\xi \in \Lambda_{(\mu, v)}^{I}(x)$. Then there exists a set $M=$ $\left\{m_{1}<m_{2}<\cdots\right\} \subset N$ such that $M \notin I$ and $(\mu, v)-\lim x_{m_{j}}=$ $\xi$.

So, given $\varepsilon>0$ and $\lambda \in(0,1)$, there exists $j_{0} \in \mathrm{N}$ such that $\mu\left(x_{m_{j}}-\xi, \varepsilon\right)>1-\lambda$ and $v\left(x_{m_{j}}-\xi, \varepsilon\right)<\lambda$, for all $\mathrm{j} \geq j_{0}$.

But the set $A=\left\{k \in N: \mu\left(x_{k}-\xi, \varepsilon\right)>1-\lambda\right.$ and $v\left(x_{k}-\xi, \varepsilon\right)<$ $\lambda\} \supset M \backslash\left\{m_{1}, m_{2}, \cdots, m_{j_{0}}\right\}$

Therefore, $A \notin I$ and hence $\xi \in \Gamma_{(\mu, v)}^{I}(x)$.

Theorem 4.5. Let $(X, \mu, v, *, o)$ be a IFNLS. Then for any sequence $x \in X, \Gamma_{(\mu, v)}^{I}(x) \subseteq L_{(\mu, v)}(x)$.

Proof. Let $\gamma \in \Gamma_{(\mu, v)}^{I}(x)$. Then for each $\varepsilon>0$ and $\lambda \in(0,1)$, the set

$\left\{k \in N: \mu\left(x_{k}-\gamma, \varepsilon\right)>1-\lambda\right.$ and $\left.v\left(x_{k}-\gamma, \varepsilon\right)<\lambda\right\} \notin I$.

We take the set $\left\{m_{1}<m_{2}<\ldots\right\} \notin I$ such that

$K=\left\{m_{j} \in N: \mu\left(x_{m_{j}}-\gamma, \varepsilon\right)>1-\lambda\right.$ and $\left.\nu\left(x_{m_{j}}-\gamma, \varepsilon\right)<\lambda\right\}$ $\notin I$, for every $\varepsilon>0$.

Since there are infinitely many elements in $K$, we have $\gamma \in L_{(\mu, v)}(x)$ and so $\Gamma_{(\mu, v)}^{I}(x) \subseteq L_{(\mu, v)}(x)$.

Theorem 4.6. In a IFNLS $(X, \mu, v, *, o)$ the set $\Gamma_{(\mu, v)}^{I}(x)$ is closed for each sequence $x=\left(x_{k}\right)$ in $X$.

Proof. Let $y$ be a limit point of $\Gamma_{(\mu, v)}^{I}(x)$. Let $t>0$ and $0<r$ $<1$. Then every open ball $B(y, r, t)=\{x \in X: \mu(y-x, t)>1-r$ and $v(y-x, t)<r\}$ with centre $y$ and radius $r$ must contain a point of $\Gamma_{(\mu, v)}^{I}(x)$ different from $y$.

Let $y_{0} \in \Gamma_{(\mu, v)}^{I}(x) \cap B(y, r, t)$.

Choose $\delta>0$ such that $B\left(y_{0}, \delta, t\right) \subset B(y, r, t)$.

Then we have,

$$
\left\{k \in N: \mu\left(y-x_{k}, t\right)>1-r \text { and } v\left(y-x_{k}, t\right)<r\right\}
$$


$\supset\left\{k \in N: \mu\left(y_{0}-x_{k}, t\right)>1-\delta\right.$ and $\left.v\left(y_{0}-x_{k}, t\right)<\delta\right\} \notin I$. Hence $y \in \Gamma_{(\mu, v)}^{I}(x)$.

Theorem 4.7. Let $(X, \mu, v, *, o)$ be a IFNLS. For a sequence $x$ $=\left(x_{k}\right)$ in $X$ if $I_{(\mu, v)}-\lim x=x_{0}$, then $\Lambda_{(\mu, v)}^{I}(x)=\Gamma_{(\mu, v)}^{I}$ $(x)=\left\{x_{0}\right\}$.

Proof. First we show $\Lambda_{(\mu, v)}^{I}(x)=\left\{x_{0}\right\}$. Let us assume that $\Lambda_{(\mu, v)}^{I}(x)=\left\{x_{0}, y_{0}\right\}$ such that $x_{0} \neq y_{0}$. Then there exists two sets $M=\left\{m_{1}<m_{2}<\cdots\right\} \subset N$ and $L=\left\{l_{1}<l_{2}<\cdots\right.$ \}$\subset N$ such that $M, L \notin I$ and $(\mu, v)-\lim x_{m_{j}}=x_{0}$, $(\mu, v)-\lim x_{l_{i}}=y_{0}$.

Therefore, for every $\varepsilon>0$ and $\lambda \in(0,1)$, we have, $\left\{l_{i} \in L: \mu\left(x_{l_{i}}-y_{0}, \varepsilon\right) \leq 1-\lambda\right.$ or $\left.v\left(x_{l_{i}}-y_{0}, \varepsilon\right) \geq \lambda\right\} \in I$. Then, we observe that $\left\{l_{i} \in L: i \in N\right\}$ $=\left\{l_{i} \in L: \mu\left(x_{l_{i}}-y_{0}, \varepsilon\right)>1-\lambda\right.$ and $\left.v\left(x_{l_{i}}-y_{0}, \varepsilon\right)<\lambda\right\} \cup$ $\left\{l_{i} \in L: \mu\left(x_{l_{i}}-y_{0}, \varepsilon\right) \leq 1-\lambda\right.$ or $\left.v\left(x_{l_{i}}-y_{0}, \varepsilon\right) \geq \lambda\right\}$ which implies that $\left\{l_{i} \in L: \mu\left(x_{l_{i}}-y_{0}, \varepsilon\right)>1-\lambda\right.$ and $\left.v\left(x_{l_{i}}-y_{0}, \varepsilon\right)<\lambda\right\} \notin I$

But as $I_{(\mu, v)}-\lim x=x_{0}$, we have

$\left\{k \in N: \mu\left(x_{k}-x_{0}, \varepsilon\right) \leq 1-\lambda\right.$ or $\left.v\left(x_{k}-x_{0}, \varepsilon\right) \geq \lambda\right\} \in I$, for all $\varepsilon>0$

Therefore,

$\left\{k \in N: \mu\left(x_{k}-x_{0}, \varepsilon\right)>1-\lambda\right.$ and $\left.v\left(x_{k}-x_{0}, \varepsilon\right)<\lambda\right\} \notin I$.

Now for every $x_{0} \neq y_{0}$, we have,

$\left\{l_{i} \in L: \mu\left(x_{l_{i}}-y_{0}, \varepsilon\right)>1-\lambda\right.$ and $\left.v\left(x_{l_{i}}-y_{0}, \varepsilon\right)<\lambda\right\}$

$\cap\left\{k \in N: \mu\left(x_{k}-x_{0}, \varepsilon\right)>1-\lambda\right.$ and $\left.v\left(x_{k}-x_{0}, \varepsilon\right)<\lambda\right\}=\phi$.

Hence,

$\left\{l_{i} \in L: \mu\left(x_{l_{i}}-y_{0}, \varepsilon\right)>1-\lambda\right.$ and $\left.v\left(x_{l_{i}}-y_{0}, \varepsilon\right)<\lambda\right\}$

$\subseteq\left\{k \in N: \mu\left(x_{k}-x_{0}, \varepsilon\right) \leq 1-\lambda\right.$ or $\left.v\left(x_{k}-x_{0}, \varepsilon\right) \geq \lambda\right\} \in I$,

which contradicts (10) and so $\Lambda_{(\mu, \nu)}^{I}(x)=\left\{x_{0}\right\}$.
Next, we assume that $\Gamma_{(\mu, v)}^{I}(x)=\left\{x_{0}, z_{0}\right\}$ such that $x_{0} \neq$ $z_{0}$. Then,

$\left\{k \in N: \mu\left(x_{k}-z_{0}, \varepsilon\right)>1-\lambda\right.$ and $\left.v\left(x_{k}-z_{0}, \varepsilon\right)<\lambda\right\} \notin I$.

Since,

$\left\{k \in N: \mu\left(x_{k}-x_{0}, \varepsilon\right)>1-\lambda\right.$ and $\left.v\left(x_{k}-x_{0}, \varepsilon\right)<\lambda\right\} \cap\{k \in N$ $: \mu\left(x_{k}-z_{0}, \varepsilon\right)>1-\lambda$ and $\left.v\left(x_{k}-z_{0}, \varepsilon\right)<\lambda\right\}=\phi$, for every $x_{0}$ $\neq z_{0}$, we have,

$\left\{k \in N: \mu\left(x_{k}-x_{0}, \varepsilon\right) \leq 1-\lambda\right.$ or $\left.v\left(x_{k}-x_{0}, \varepsilon\right) \geq \lambda\right\}$

$\supseteq\left\{k \in N: \mu\left(x_{k}-z_{0}, \varepsilon\right) \leq 1-\lambda\right.$ or $\left.v\left(x_{k}-z_{0}, \varepsilon\right) \geq \lambda\right\}$

From (12) the R.H.S. of (13) does not belong to $I$ and from (11) the L.H.S. of (13) belongs to $I$, which is a contradiction. Hence, $\Gamma_{(\mu, v)}^{I}(x)=\left\{x_{0}\right\}$.

\section{CONCLUSION}

In this paper we have introduced the concept of I-limit superior and $I$-limit inferior for sequences in IFNLS. Also the concept of $I$-limit points and $I$-cluster points in IFNLS has been introduced and studied.

\section{ACKNOWLEDGMENTS}

The authors thank the reviewers for their comments and suggestions.

\section{REFERENCES}

[1] Anastassiou, G. A. 2004. Fuzzy approximation by fuzzy convolution type operators. Comput. Math. Appl. 48,1369-1386.

[2] Atanassov, K. T. 1986. Intuitionistic fuzzy sets. Fuzzy Sets. Syst. 20,87-96.

[3] Demirci, K. 2001. I-limit superior and I-limit inferior, Math. Communications. 6(2), 165-172.

[4] Erceg, M. A. 1979. Metric spaces in fuzzy set theory. J. Math. Anal. Appl. 69, 205-230.

[5] Jäger, G. 2000. Fuzzy uniform convergence and equicontinuity. Fuzzy Sets Syst. 109,187-198.

[6] Kostyrko, P., Šalát, T. and Wilczyński, W. 2000. Iconvergence. Real Anal. Exchange. 26, 669-686.

[7] Saadati, R., Park, J. H. 2006. Intuitionistic fuzzy Euclidean normed spaces. Commun. Math. Anal. 12,8590

[8] Schweizer, B., Sklar, A. 1960. Statistical metric spaces. Pacific J. Math.10, 314-344.

[9] Zadeh, L. A. 1965. Fuzzy sets. Inform. Cont. 8 , 338-353. 